\title{
Young man presenting with clubbing - A case-based evaluation
}

\author{
Herath $\mathrm{YB}^{1}$, Indrakumar J2 , Matthias $\mathrm{AT}^{3}$
}

\begin{abstract}
Clubbing is the bulbous enlargement of the distal segment of digits. It is known to be associated with several cardiological, gastrointestinal, pulmonary, neoplastic, infectious, and endocrinological disorders. Rarely clubbing may not associate with any underlying pathology despite presenting in a later stage. Here we report a case of a 26- year- old man who presented with a history of recent onset of clubbing. Following extensive evaluation we were unable to find a possible etiology.
\end{abstract}

Keywords - Idiopathic Clubbing, Hippocrates fingers, Clubbing

\section{Introduction}

Digital clubbing was first described by Hippocrates nearly 2500 years ago. It is the bulbous enlargement of the distal segment of digits. ${ }^{1}$ The proliferation of connective tissue between the nail matrix and the distal phalanx results in increase of the nail diameter. Clubbing is known to be associated with several cardiological, gastrointestinal, pulmonary, neoplastic, infectious, and endocrinological disorders. Some of the common conditions associated with clubbing are, bronchogenic carcinoma, bronchiectasis, lung abscess, cyanotic heart disease, sub-acute infective endocarditis, cirrhosis and inflammatory bowel disease. ${ }^{2}$ Clubbing may be idiopathic which is described as a rare occurrence in literature. ${ }^{3}$

\section{Case report}

A 26- year- old man was admitted to the Colombo South Teaching Hospital medical ward with a history of gradually progressing swelling of the terminal part of all fingers and toes for 5 years duration. He was clinically well and there was no history of chest pain, difficulty in breathing or limitation of physical activities, syncope, cyanosis, cough or any gastrointestinal

\footnotetext{
${ }^{1}$ Registrar in Internal Medicine, Colombo South Teaching Hospital Professorial Unit

${ }^{2}$ Senior Professor and Head, Department of Medicine, University of Sri Jayewardenepura, Specialist Physician University Medical Unit Colombo South

Teaching Hospital

${ }^{3}$ Senior Lecturer, Department of Medicine, University of Sri Jayewardenepura, Specialist Physician, University Medical Unit Colombo South Teaching Hospital

Corresponding Author: Yanushka Herath, 59, Polhengoda road, Colombo 5 yanushkaherath@gmail.com
}

symptoms. He had no previous history of significant Ilnesses except for a history of being treated for contact dermatitis. There was no family history of clubbing or any chronic illness. He has been a non-smoker and consumed alcohol only occasionally. There was no exposure to toxins. He did not use cannabis or any illicit substances.

On examination he had advanced clubbing in all the fingers and toes.(Figure -1) There were no lymphadenopathy, pallor joint swelling, joint tenderness or icterus. Blood pressure was $130 / 80 \mathrm{mmHg}$ and pulse rate $88 \mathrm{bpm}$. There was no thickening of skin over arms and legs and abdominal examination, did not show any hepatosplenomegaly. Examination of the chest was normal and no murmurs were audible. Optic fundus was normal.

His laboratory results showed a leukocyte count of $8 \times 109 / \mathrm{L}$ with a platelet count of $286 \times 109 / \mathrm{L}$ and the hemoglobin was $13.5 \mathrm{~g} / \mathrm{dL}$. His aspartate aminotransferase level was $37 \mathrm{U} / \mathrm{I}$ and alanine aminotransferase was $33 \mathrm{U} / \mathrm{L}$. His serum bilirubin level was $19 \mathrm{mmol} / \mathrm{L}$ and alkaline phosphatase level was $190 \mathrm{U} / \mathrm{L}$.

The serum creatinine was 91 micromoles/L on admission with a potassium of $3.8 \mathrm{mmol} / \mathrm{L}$ and sodium of $137 \mathrm{mmol} / \mathrm{L}$ His C-reactive protein was $<5 \mathrm{mg} / \mathrm{dl}$. Further his chest radiograph, electrocardiogram, 2D Echocardiography, abdominal ultrasound scan and High-resolution computed tomography of chest were also normal. There were no features of periostitis or new bone formation seen in the hand $x$-rays.(Figure -2) Arterial blood gases were within normal range. Retroviral studies were negative. Hepatitis B s Ag and Hepatitis $C$ antibodies were negative. Serum calcium was $2.3 \mathrm{mmol} / \mathrm{L}$. In the absence of a known aetiology a diagnosis of idiopathic clubbing was made and the patient was reassured. 


\section{Discussion}

Clubbing may occur bilaterally or unilaterally and may involve both upper or lower limbs. It may be primary or secondary. Primary clubbing can be divided as idiopathic, hereditary or associated with pachydermoperiostosis. Secondary clubbing may be due to a wide spectrum of diseases of which lung diseases accounts for $75-80 \%$ while cardiac conditions account for $10-15 \% .{ }^{4}$ Hereditary clubbing occurs in the childhood in the absence of an underlying condition.

In this patient we have not found a known cause for clubbing. He did not have any other family member or immediate relative who had clubbing. Neither he

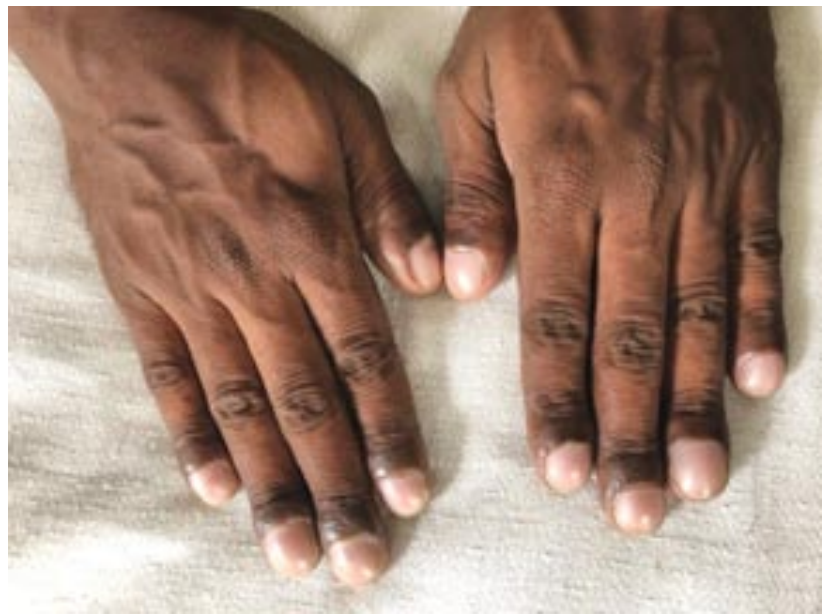

Figure 1 - Stage 4 Clubbing in bilateral hands

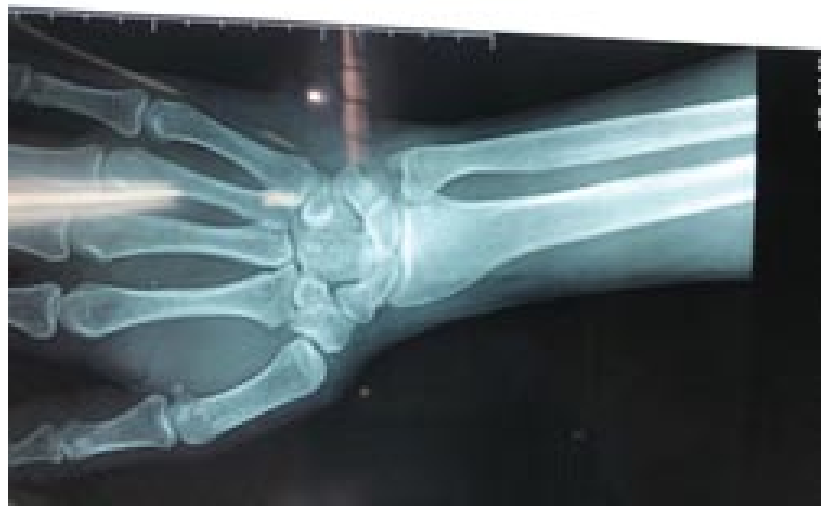

Figure 2- $X$ ray of Right side wrist joint showing no evidence of periostitis or new bone formation

did not have any facial skin thickening nor excessive sweating to suggest pachydermoperiostosis. Idiopathic clubbing is rare but reported. In the previously reported limited number of idiopathic clubbing cases such as Buchman (1956) it was tabled as such entity after extensively investigating for a possible etiology. 5
Although many possible mechanisms are proposed a definite mechanism for clubbing is still unknown. Shneerson (1981)describes the most likely mechanisms for clubbing as, vasodilatation, tissue hypoxia, neuro-circulatory reflexes, and genetic factors. ${ }^{6}$ Possible systemic vasodilatory factors causing clubbing are believed to be ferritin, bradykinins, prostaglandins, adenine nucleotides, and 5- hydroxytryptamines. ${ }^{7}$ Furthermore platelet derived growth factors and vascular endothelial growth factors which are released by platelet clumps in digital vessels are also known to play a role in the pathogenesis of finger clubbing.

\section{Conclusion}

Idiopathic clubbing is usually identified by the clinician in routine clinical examination when a patient presents for a non-related condition or more rarely presents with clubbing itself as in this patient. However, it is possible that secondary clubbing may occur even before the manifestation of an underlying disease. Therefore, it is important to follow up the patient for a period of time in order to exclude the possibility of an illness which may occur subsequently. ${ }^{8}$ However as this patient has had clubbing for five years it is unlikely that he may develop a serious underlying illness or hypertrophicosteoarthropathy in the future.

\section{References}

1. Bigler, F. C. "The Morphology of Clubbing." The American Journal of Pathology, 1958.

2. Marrie, Thomas J., and Neil Brown. "Clubbing of the Digits." American Journal of Medicine, 2007.

3. Peerbhoy MS, Rajan KE, Deoskar RB, Barthwal MS. Idiopathic clubbing [3]. Journal of Association of Physicians of India. 2006.

4. Chan CW. Evaluation of digital clubbing. Aust Fam Physician. 2015; Mar;44(3):113-6.

5. Buchman, Delbert, Edward A H. "Idiopathic Clubbing And Hypertrophic Osteoarthropathy." A.M.A. Archives of Internal Medicine 97, no. 3 (March 1, 1956): 355-58.

6. Shneerson JM. Digital clubbing and hypertrophic osteoarthropathy: The underlying mechanisms. British Journal of Diseases of the Chest. 1981 Apr;75(2):113-31.

7. Dubrey S, Pal S, Singh S, Karagiannis G. Digital clubbing: Forms, associations and pathophysiology. British Journal of Hospital Medicine. 2016.

8. Vandemergel, X., and B. Renneboog. "Prevalence, Aetiologies and Significance of Clubbing in a Department of General Internal Medicine." European Journal of Internal Medicine, 2008. 